\title{
Reichenbach and Weyl on apriority and mathematical applicability
}

\author{
Sandy Berkovski
}

Received: 13 May 2009 / Accepted: 8 June 2009 / Published online: 30 June 2009

(C) Springer Science+Business Media B.V. 2009

\begin{abstract}
I examine Reichenbach's theory of relative a priori and Michael Friedman's interpretation of it. I argue that Reichenbach's view remains at bottom conventionalist and that one issue which separates Reichenbach's account from Kant's apriorism is the problem of mathematical applicability. I then discuss Hermann Weyl's theory of blank forms which in many ways runs parallel to the theory of relative a priori. I argue that it is capable of dealing with the problem of applicability, but with a cost.
\end{abstract}

Keywords Relative a priori $\cdot$ Convention $\cdot$ Reichenbach $\cdot$ Weyl $\cdot$ Friedman

\section{Introduction}

A view widespread already in the early 1900s was to see laws of nature as conventions. A popular example was the statement:

Phosphorus melts at $44^{\circ} \mathrm{C}$.

The melting temperature is not a sufficient characteristic, but a necessary one. Suppose there is a given solid substance $X$. To verify whether $X$ is phosphorus we heat it to $44^{\circ} \mathrm{C}$ and observe its behaviour. If it starts melting, there are other tests to perform for establishing the nature of $X$. If it fails, we raise a hypothesis that $X$ is not in fact phosphorus.

Far from believing in the essential attributes of phosphorus that scientists uncover in the course of their experiments, many theorists believed that the statement (1) contributes to the way we understand the term 'phosphorus'. Empirical regularities

\footnotetext{
S. Berkovski $(\varangle)$

Department of Philosophy, Bilkent University, FA Building, 06800 Ankara, Turkey

e-mail: sandyber@bilkent.edu.tr; sandy.berkovski@gmail.com
} 
observable in experiments affect the way we talk about material substances and the way we describe the experimental outcome. If such is the role of laws, they become akin to conventions, viz. arbitrary stipulations. I stipulate the term 'phosphorus' to refer to the substance melting at $44^{\circ} \mathrm{C}$ (plus a set of other characteristics), but there is nothing wrong with you if you decide to stipulate the same term to refer to something melting at $45^{\circ} \mathrm{C}$. All we have to do is to find a way to correlate our respective linguistic habits. There is nothing wrong with either of those habits, since there is nothing in the string of noises and shapes ' $\mathrm{p} \neg \mathrm{h} \frown \mathrm{O}^{\frown} \frown \mathrm{s} \frown \mathrm{p} \frown \mathrm{h} \frown \mathrm{o} \frown \mathrm{r} \frown \mathrm{u} \frown \mathrm{s}$ ' itself which singles out the correct way of using it.

The parallel between laws and ordinary linguistic conventions is obvious: natural languages are likewise the products of history, a result of explicit and implicit conventions multiplied since time immemorial. Being conventions, laws are neither true, nor false. Statements about conventions can be true or false, and similarly statements $u$ sing those conventions. The conventionalist view, in the form outlined here, is associated with the name of Poincaré. Although Poincaré's concern is geometry, it is thought that conventionalism about geometry easily extends to conventionalism about physical sciences. The common perception is reinforced by his explicit appeal to the analogy with natural languages. Thus in Chap. III of Poincaré (1952) we find a little dictionary allowing mutual translation of statements of Lobatchevsky's and Euclidean geometry. The common perception I am talking about is in point of fact misleading. Michael Friedman contributed significantly to clarifying Poincaré's real position, and I shall say a few words about that below.

Even if we deny the conventional character of natural laws, one may be reluctant to treat them as mere generalisations of observational data. That is, one may deny that laws are both empirical and contingent. In recent years this point has been stressed by Kripke's theory metaphysical necessity elaborated in Lecture III of Kripke (1971, 1980). Kripke attributed metaphysical necessity to 'theoretical identifications' exactly of the sort displayed by the statement (1). Some theorists subsequently sought to eliminate the gap between metaphysical necessity and physical necessity. Metaphysically necessary statements receive their elevated modal status from certain very general physical laws which govern theoretical identifications. What is particularly important to us here is that Kripke regards the source of metaphysical necessity as being a priori. If we now identify metaphysical necessity with physical necessity of a special kind, then those general physical laws will in turn be known a priori.

As we all know, there is a venerable tradition, according to which laws of nature are indeed known a priori. Descartes, Spinoza, and Leibniz should be counted among its adherents despite significant and subtle differences between them. However, with the advent of empiricism and naturalism the apriorist tradition has fallen out of favour. The most sustained recent attempt to resuscitate it was made by Michael Friedman. His studies of Kant's philosophy of science showed that Kant, for better and for worse, was part of that same tradition. If the first Critique is concerned chiefly with the apriority of categories and pure intuition and tends to obscure Kant's stance on natural laws, even a cursory perusal of Kant (1996) makes it plain:

What can be called proper science is only that whose certainty is apodictic; cognition that can contain mere empirical certainty is only knowledge improperly 
so-called. ... A rational doctrine of nature thus deserves the name of a natural science, only in case the fundamental natural laws therein are cognised a priori, and are not mere laws of experience. etc. etc. (Kant 1996, p. 468)

Friedman's ambition did not stop at merely making an historical correction vis-àvis Kant. His latest enquiries pursue a further twofold goal. One part of it consists in showing the continuity between Kant's thought and the Vienna Circle, including Reichenbach (who resided in Berlin). That part of the project was started in Friedman (1983) and articulated in Friedman (1999). The second part of the project is less historical. In Friedman (2001) it is argued that a version of the aprioristic conception is the correct view on the nature of scientific theories. Furthermore, an aprioristic correction of positivism would gain us a more balanced and plausible interpretation of Kuhn's historical data, as well as highlight the pitfalls of Quinean holism.

In what follows I shall critically review Friedman's interpretation of Reichenbach (1965). The main focus here will be on the notion of relative a priori and the tripartite distinction of physical sciences. I shall then discuss an alternative view on the same stock of issues due to Hermann Weyl.

\section{Reichenbach on the relative a priori}

The Kantian notion of synthetic a priori was thought to be compromised by the development of non-Euclidean geometries. Whereas Kant claimed that the Euclidean geometry is a priori true, the development of non-Euclidean geometries showed that it is not to be believed a priori. The general theory of relativity (GTR) then showed that it is not even true. Such was the reasoning prominent in the late 1910s. Reichenbach resists it in Reichenbach (1965). His argument turns on distinguishing two connotations of a priori in Kant. One such connotation relates to eternal truth, to being 'true for all times'. That is, $S$ is a priori true just in case $S$ is true at all times. This is an extraordinary view, exegetically and in content. I do not have the German source handy here, but I trust that the translation got it right. The view is remarkable, because Reichenbach's Kant is made committed to believing that statements, or judgements, are true at some times, and false at other. This is a familiar medieval conception, perhaps also attributable to Aristotle; but I doubt very much that Kant has ever expressed sympathy with it.

However, when we look at the textual evidence that Reichenbach gives in the footnote 17 , we realise that no revolutionary exegesis is at stake. The first connotation of a priori equates it with strict universality. That is, $S$ is a priori true just in case $S$ is true in all possible circumstances. That formulation makes a link between apriority and necessity. It also does not distinguish between synthetic apriority and analytic apriority. ${ }^{1}$ The second connotation of a priori which Reichenbach attributes to Kant relates to the construction of objects of experience. Our experience obeys certain rules of organisation. The sensibility and the reason of the agent contribute to the way we perceive the world, and they do that in accordance with rules. Therefore, within this connotation, $S$ is a priori just in case the truth-value of $S$ is established solely through those 'constitutive' rules of experience. Reichenbach does not provide any textual

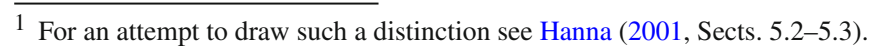


evidence for his second connotation, because, as he says, it 'will not be disputed'. He refers us to the Transcendental Deduction of the first Critique.

Now, Reichenbach's idea is to discard the first connotation and to keep the second one. Kantian rules that we have just mentioned are labelled as 'axioms of coordination' (synonymous with 'coordinating principles'). Their role is in essence to control the way we reason about observational data. Says Reichenbach:

The reality of things must be distinguished from the reality of concepts which, insofar as one wishes to call them real, have a mere psychological existence. But there remains a strange relation between the real thing and the concept, because only the coordination of the concept defines the individual thing in the "continuum" of reality; and only the conceptual connection decides on the basis of perceptions whether a conceived individual thing "is there in reality". (Reichenbach 1965, pp. 50-51)

This is the situation, according to Reichenbach, in analytic geometry. There we have algebraic representations of geometrical concepts. Thus, upon fixing the coordinate cross we can determine a one-parameter family of curves by the algebraic function $f(x, y, z)=0$. In the case of Euclidean geometry, such a principle is embodied in the Euclidean metric which fixes the relation which is to be obtained for a collection of spatial points to form a spatially extended body. Similarly, we may coordinate certain mathematical symbols for vectors with physical forces and thereby conceive the latter as objects having vector-like properties.

By discarding the first connotation of a priori we maintain that the Euclidean geometry is not necessarily, or universally, true. What does that mean? Here, I think, Reichenbach's reasoning becomes fairly difficult to understand. On one hand, Reichenbach interprets apriority qua necessity and universality as immunity to revision. ${ }^{2}$ On the other hand, he effectively claims that revisability of coordinating principles does not amount to their 'total' falsehood. They should never be abandoned in their entirety as we might abandon a revised, viz. false, generalisation. Rather, we should always seek to replace them with those principles which differ from them only with respect to a limited number of instances of the available data. Reichenbach elaborates in the following key passage:

Until now all results of physics have been obtained by means of the self-evident system. We discovered that this fact does not exclude a contradiction the existence of which can be ascertained-but how shall we obtain a new system? With respect to individual laws, this aim is easily reached because only those presuppositions that contain the individual law have to be changed. But we have seen that all laws contain coordinating principles, and if we wish to test new coordinating principles inductively, we must first change every physical law. It would indeed be nonsensical to test new principles by means of experiences still presupposing the old principles. If, for instance, space were tentatively assumed to be four-dimensional, to test the assumption, all methods of measuring lengths used until now would have to be abandoned and to be replaced

$\overline{2}$ See Reichenbach (1965, p. 55). 
by a measurement compatible with four-dimensionality. Furthermore, all laws concerning the behavior of the material used in the measuring instrument, concerning the velocity of light, and so forth, would have to be given up. Such a procedure would be technically impossible. We cannot start physics all over again. (Reichenbach 1965, pp. 67-68)

Interestingly, Reichenbach gives only pragmatic reasons for the 'lax' replacement of the old coordinating principles. The old principles are nothing but inductive approximations of the new ones. We, therefore, get a familiar chart of the cumulative development of science, in which theories - as time goes by- 'improve', whether that means accounting for more and more phenomena or getting closer and closer to truth (although the cumulative chart might not require postulating any one single unreachable true theory). This is a view current among practicing scientists. Let us call it 'the Folk View'. Kepler's laws, according to that view, are regarded as approximations of Newton's laws. The laws of stationary electric and magnetic fields are regarded as approximations of Maxwell's equations. Some of these will no doubt qualify as what Reichenbach terms 'axioms of connection', that is, ordinary physical laws which are mere generalisations from experience. But of course the Folk View respects no sharp distinction between axioms of connection and axioms of coordination. For instance, a typical authoritative textbook tells us:

Subatomic particles behave in a more complex way than the material points of the classical mechanics. The classical picture only approximately reflects the laws of nature. (Sivoukhin 1979, Sect. 5)

Newton's theory of gravitation was further developed in Einstein's general theory of relativity. This latter gives not an intuitive explanation of gravitation, but a new way of describing it and a generalisation of Newton's theory. (Sivoukhin 1979, Sects. 55-57)

The theory of relativity and quantum mechanics are more general theories than Newtonian mechanics. The latter is contained in them as an approximate limiting instance. Relativistic mechanics merges into Newtonian mechanics in the case of low velocities. Quantum mechanics merges into Newtonian mechanics in the case of sufficiently massive bodies moving in smoothly varying fields. (Sivoukhin 1979, Sects. 0-5; italics added)

According to the Folk View, then, the new principles extend the area of application of the old principles. The replacement of old principles is nothing but generalisation. Perhaps we should better talk about old principles being 'superseded' by new ones.

Now, Reichenbach's view amounts to the introduction of different standards of revision. Empirical laws, or the axioms of connection, may be abandoned in the course of scientific development as a result of obtaining new evidence. Coordinating principles of a given theory are those statements of the theory which are superseded, rather than completely abandoned, by their successors. However, Reichenbach supplies no systematic reason for thinking that there is a sharp distinction between two families of principles. The distinction appears to be based on a practical impossibility. Therefore, I think, Reichenbach's conception of the relative a priori resembles the Folk View more closely than one might expect: the latter equally insists on incorporating 
the fundamental laws of the earlier theories into the novel ones. ${ }^{3}$ The earlier laws are approximations, or limiting cases, of the novel laws. We shall see in a moment that this resemblance is not accidental.

\section{Friedman's programme}

Quinean holism regards all individual beliefs as being inserted in a network. Abandoning one belief impinges on holding other beliefs in the network. To borrow John Carriero's apt metaphor, it is as if the web of belief is composed of infinitely viscous fluid-like matter where the smallest local change reverberates throughout its farthest reaches. Therefore, no principled distinction between the a priori and the empirical is possible. If revising one belief on empirical grounds forces an adjustment of the whole network, logical and mathematical beliefs would also appear empirical.

And holistic explanations seem congruent with Kuhn's picture of scientific change. Since statements are never to be checked individually, but only as part of 'theories', those theories cannot be compatible. We can expand the notion of theory to include standards of experimentation, relevant textbooks, and so forth. We will then obtain Kuhn's notion of structural lexicon. Projecting the resulting view on the history of a particular discipline, we will get a sequence of isolated paradigms eliminating each other without a trace.

Friedman resists both Quinean holism and Kuhn's picture of scientific change. ${ }^{4}$ His programme laid out in Friedman (2001) has many aspects, but these two of them seem to me the most important ones. More specifically, his claims are as follows:

Contra Quine: historical developments in mathematical physics show that there is a hierarchy of beliefs. Mathematical beliefs are a priori and properly physical are empirical. There is a third class of beliefs characterised as relative a priori which mediates between the mathematical and the physical parts of the theory. Contra Kuhn: historical developments in mathematical physics show that there is far more continuity in successive paradigms than Kuhn allows. The fact of continuity is to be explained by the class of relative a priori beliefs.

I will have little to say about Friedman's polemic with Kuhn. As far as I can tell, Friedman happily endorses the Folk View in the outline. However, an important insight of Kuhn's is preserved in that the a priori framework of an earlier theory cannot accommodate the framework of the later one. It is only in a retrospect, from a historical point of view, when we are already in possession of a later, 'expanded' theoretical framework, that we make sense of the earlier one. ${ }^{5}$

I will predominantly focus on the notion of relative a priori. Friedman derives it from Reichenbach (1965). The historical claim here is that before the logical positivists, and in particular Schlick and Reichenbach, adopted a thoroughly conventionalist outlook, they toyed with the idea of adapting the Kantian a priori to the development

\footnotetext{
3 Coffa draws a similar conclusion: see Coffa (1991, p. 203).

4 See also Friedman (2001).

5 See Friedman (2001, 63ff, 96ff). For a criticism see van Fraassen (2006, pp. 300-303).
} 
of relativistic mechanics. The result is a tripartite division of statements of physical theory. Apart from mathematical and empirical statements we also have a third class of relative a priori statements. They comprise mechanics which is sharply distinguished from the main body of physics.

In Friedman's hands, Reichenbach navigates skillfully between orthodox Kantianism, Poincaré's conventionalism, and Helmholtzean empiricism. Relativised a priori is not a Kantian a priori, since there is a way in which it is revisable. It is, on the other hand, not a mere empirical law, since it occupies a different place in the hierarchy of our knowledge. Not only it is never fully abandoned, but also the scope of admissible evidence depends on which principles are accepted as relative a priori. For instance, classical mechanics allows absolute contraction, and therefore, treats length as an absolute property of bodies. Not so in special relativity, where length becomes dependent on the frame of reference. That relativisation of length in Lorentz transformations is very clearly not an empirical generalisation.

Yet, why should one insist on regarding it as an a priori principle, and not a convention? In many ways Poincaré's conventionalism occupies middle ground in the debate over the synthetic a priori. A convention does not reflect a property of reality, at least not literally, nor does it reflect our capacities allowing us to gain knowledge prior to all experience. Conversely, it is not an empirical statement: it would hardly be intelligible to try to test a convention by observation. If Reichenbach could successfully differentiate himself from Poincaré, he would be able to defend a second alternative position intermediate between apriorism and empiricism. From a historical perspective, too, the issue holds special importance for understanding Schlick's criticism of Reichenbach's 1920 book. Schlick interpreted the relative a priori as a form of convention. Reichenbach eventually came to accept Schlick's criticism and endorsed conventionalism, albeit with qualifications. ${ }^{6}$ If a second intermediate position is available, Schlick would have misinterpreted Reichenbach, while the latter would have failed to see the novelty of his own approach.

\section{The ghost of convention}

One key distinction between the relative a priori and the empirical lies in the way they are revisable. Empirical generalisations can be subjected to experimental tests and can be retained or abandoned depending on the results of those tests. Relative a priori statements behave differently. Consider the familiar story of the light principle of special relativity. ${ }^{7}$ It is not established by the Michelson-Morley experiment. The latter does not show the constant velocity of light in all inertial frames: it only shows that there are no identifiable differences in the velocity of light in different frames. Lorentz's electrodynamics, based on Newtonian mechanics, was developed with the express purpose of accommodating the results of the experiment. Special relativity

\footnotetext{
6 See e.g. Reichenbach (1958, pp. 35-36). The Schlick-Reichenbach exchange is discussed in Coffa (1991, pp. 202-203) and Friedman (1999, pp. 62-68).

7 See Friedman (2001, pp. 87-88).
} 
makes, therefore, a leap, unsupported by empirical input of the Michelson-Morley, when it postulates the light principle. Einstein uses it to define simultaneity and the new metric of the spatiotemporal framework. The Newtonian framework and the associated Euclidean metric were unseated as a result, not of an experimentum crucis, but of Einstein's postulation.

A Quinean holist reads this story as being essentially incomplete. True, there is no one experiment that would serve as a tiebreaker between alternative metrics. But there were multiple empirical results, notably in electrodynamics, that motivated the search for a non-Euclidean metric. The apriorist's rejoinder is swift. Whatever motivation there was, it is at best a biographical episode. It is not sufficient to justify the choice of the new metric. The crucial factor involved in that choice was not coming from empirical testing.

It is though much less clear how an apriorist fares against a conventionalist. From the introduction of the Minkowskian metric the conventionalist is happy to learn his conventionalist lesson: the light principle was a convention. Friedman and Friedman's Reichenbach insist that it is an a priori statement. What is really at stake here beyond the terminological distinction? In one sense, the answer is relatively straightforward and uncomplicated. As Reichenbach notes in the very beginning of Reichenbach (1965), general relativity refuted Poincaré's conventionalism. It showed that Euclidean geometry no longer represents the geometry of physical space-time. The curvature depends on the distribution of matter. And since the metric is chosen on the basis of physical considerations, geometry is transformed into a properly empirical science.

But it is not a very satisfactory response. For it deals with a narrow conception of conventionalism which applies to geometry. One can extend its application to every theory. The epistemic status of the claims - empirical or conventional-is not permanent. They may be conventional in one theory, but regarded empirical in another. So whilst in Newtonian mechanics geometry was conventional, in GTR it becomes empirical. What remains conventional in it is, for example, the global topology of the universe.

Now, that is precisely Friedman's conception of 'dynamic a priori' ${ }^{8}$ The only difference, of course, is that the non-empirical, non-inductive, changeable level is called 'a priori', rather than 'conventional'. A question arises about what exactly the gains of that shift in terminology should be. As far as I can tell, the point remains obscure. Whenever Friedman discusses Poincaré's work, the emphasis is put always on the advances of GTR of which Poincaré was unaware, thus echoing Reichenbach's own misgivings expressed in the letter to Schlick. ${ }^{9}$ But when he comes to examine Carnap's version of conventionalism, he is prepared to identify Carnap's 'conventional' L-rules from The Logical Syntax of Language with the relativised a priori. Indeed, he remarks that 'Carnap articulates a version of Poincaré's conventionalism that is as general as possible.' 10 Schlick's and Reichenbach's own later reading of the theory presented

\footnotetext{
8 See e.g. Friedman (1999, p. 66).

9 See e.g. Friedman (1999, pp. 83-84).

10 See Friedman (1999, pp. 66-67).
} 
in Reichenbach (1965) was misleading only because they erroneously endorsed Poincaré's conventionalism about geometry. They ignored its empirical status in GTR and along with it the possibility of a dynamic a priori. The Logical Syntax of Language restores to a large extent the vision first presented in Reichenbach (1965).

Friedman's reading of Reichenbach leads us, therefore, to a generalised version of conventionalism. And under a different, and Friedman's preferred, angle, we have arrived at a circumscribed version of Kantian apriorism. We no longer believe in an eternal a priori, but we do still believe in a class of assumptions, whatever their name, that determine the conception of an object for a given theory. But I wish to draw attention to a particular issue, carrying special significance for Reichenbach's and Friedman's project, which divides sharply between the original version of apriorism and its watered-down version. If arithmetic and Euclidean geometry reflect immutable forms of pure intuition and if the objects of experience are equally determined by those forms, then there is no mystery in applying mathematics in empirical enquiry. Thus physical space necessarily possesses Euclidean metric: both have the same source. Kant triumphantly concludes in the Prolegomena:

My doctrine of the ideality of space and time, therefore, so far from making the whole world of senses into mere illusion, is rather the only means of securing the application to real objects of one of the most important kinds of knowledge, namely that which mathematics expounds a priori, and of preventing it from being held to be mere illusion, because without this observation it would be quite impossible to decide whether the intuitions of space and time, which we take from no experience and which yet lie in our representations a priori, were not mere chimeras of the brain made by us to which no object corresponds, at least not adequately, and thus geometry itself a mere illusion; whereas on the contrary, just because all objects of the world of the senses are mere appearances, we have been able to show the indisputable validity of geometry in respect of them. (Kant 1953, p. 49)

A Quinean holist, someone who believes in the empirical status of mathematics, may attempt at a similar argument. Since mathematics and natural science at bottom have the same empirical source, there is no wonder why mathematical concepts are found to be useful in empirical research. But if we recognise the a priori character of mathematics, we open up a gap: we create a problem of explaining why its concepts are useful in empirical disciplines. Now a host of issues must be resolved before a proper debate begins. Perhaps one should in the first place clarify why the problem deserves any explanation at all. ${ }^{11}$ But in the framework of Reichenbachian apriorism the problem receives a neat (Kantian) formulation. Since the role of the relative a priori is in mediating between the level of mathematics and the level of empirical laws, one should ask how the relative a priori is possible. We know that the mediation takes place. But we do not know why it has been successful. Indeed, most of its success is due to the advances in mathematical physics, the primary foci of Friedman's account.

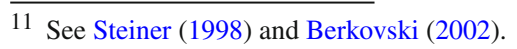


I conclude that Reichenbach and Friedman have failed in formulating the second intermediate position referred to above. What I propose to do now is to look at Weyl's views that in some aspects overlap Reichenbach's and which, moreover, were developed at exactly the same time. Weyl's account offers a solution to the problem of mathematical applicability_but with a considerable cost.

\section{Weyl on mechanics and electromagnetism}

Hermann Weyl underwent many philosophical transformations in his career. At its various stages he was associated with constructivism, conventionalism, and formalism. Platonist sentiments can occasionally be found in his writings, while Duhemian holism is a running theme there. He was under the spell of the first Critique in his youth; later on Fichte and, more significantly, Husserl were among his major influences. The unusual diversity of Weyl's philosophical beliefs was no accident. In his own words, he was like 'a bumble-bee, flying between different flowers and trying to draw a little bit of nectar from each one of them'. ${ }^{12}$ It would therefore be futile to attribute a single well-argued philosophical doctrine to Weyl. We must regard him as a mathematician and physicist of the first rate deeply sensitive to the philosophical perplexities induced by his discipline.

A particular argument to which I wish to draw attention here occurs in the discussion of stationary electromagnetism in Weyl (1922). Weyl gives a standard, if abridged, derivation of its field equations. Suppose we have two electric charges $q_{1}$ and $q_{2}$ in the given fragment of space. Coulomb's Law allows us to describe the force existing between those charges when they occupy fixed positions. The force exerted on $q_{1}$ is given by the formula:

$$
\mathbf{F}_{1}=\frac{1}{4 \pi} \frac{q_{1} q_{2}}{r_{12}^{2}} \mathbf{r}_{12}
$$

Another notion is commonly introduced, namely, the notion of electric field which is just the force exerted upon the point-charge $e$ :

$$
\mathbf{F}=e \cdot \mathbf{E}
$$

We generalise to the case of several charges. The field will be the sum of the contribution of all the charges at the given location:

$$
\mathbf{E}=\sum_{j} \frac{1}{4 \pi} \frac{q_{j}}{r_{1 j}^{2}} \mathbf{r}_{1 j}
$$

Then we take the integral on the assumption that the charges are distributed in space with the 'density' $\rho$ :

12 See Weyl (1955). 


$$
\mathbf{E}=\frac{1}{4 \pi} \int \frac{\rho \cdot \mathbf{r}}{r^{3}} d V
$$

Now, on the other hand, we can introduce the notion of electric potential computed by the formula:

$$
\phi=-\frac{1}{4 \pi} \int \frac{\rho}{r} d V,
$$

whence we get that:

$$
\mathbf{E}=-\nabla \phi=-\operatorname{grad} \phi
$$

Therefore, since $\mathbf{E}$ is a gradient of the scalar field, we have:

$$
\nabla \times \mathbf{E}=\operatorname{curl} \mathbf{E}=0 .
$$

By Gauss' Theorem we also determine that the flux of $\mathbf{E}$ from an enclosed surface is equal to the quantity of charges within that surface; therefore:

$$
\nabla \cdot \mathbf{E}=\operatorname{div} \mathbf{E}=\rho .
$$

After thus presenting a fairly standard account of electric field-reproduced here with only minor alterations-Weyl follows up with a densely argued philosophical gloss. I shall now try to unpack it. Coulomb's Law describes action at a distance. If any of the charges is moved, the force exerted by it on another charge will change accordingly. But is such a situation even intelligible? The answer must be in the negative. Coulomb's Law provides a way to compute the magnitude of force. But we should not regard it as explaining what force is. The real explanation is given by the field equations (2) and (3). And they embody a principle directly opposite to action at a distance. Combined together, they yield Coulomb's Law, not vice versa. Yet, why should we adopt this particular explanatory order? Here is Weyl:

[We] bow to the dictates of the theory of knowledge. Even Leibniz formulated the principle of continuity, of infinitely near action, as a general principle, and could not, for this reason, become reconciled to Newton's Law of Gravitation, which entails action at a distance and which corresponds fully to that of Coulomb. The mathematical clearness and the simple meaning of the laws [(2) and (3)] are additional factors to be taken into account. (Weyl 1922, p. 66)

The 'general' principle to which Weyl has resorted is most clearly not part of the physical theory itself. It is a philosophical principle and it determines the choice of the physical theory. Its precise epistemic role will have to be clarified further.

Weyl, meanwhile, continues by linking mechanics to physics. There is no separate empirical science of mechanics. The concept of force is not provided by mechanics. Newton's Second Law does not explain what force is; that is, force does not signify mass $\times$ acceleration. That is hardly a controversial view to hold. What one is expected 
to say instead is that Newton's Second Law allows merely to compute force when the values for mass and acceleration are given. That is, indeed, what Weyl appears to claim a little later. But he also insists on a different cryptic remark:

Mechanics does not, however, teach us what is force; that we learn from physics. The fundamental law of mechanics is a blank form which acquires a concrete content only when the conception of force occurring in it is filled in by physics. (Weyl 1922, pp. 66-67, his italics)

What exactly does it mean to say that $F=m \cdot a$ is a blank form? A natural interpretation would be that, so long as we are confined to mechanics, we are dealing with a mere mathematical formula devoid of physical content. I believe that we cannot appreciate the significance of this claim without putting the role of mathematical symbolism into a wider perspective.

Before we do that, let us finish with Weyl's argument. For it ends with an energetic apology for holism. There is a network of laws which cannot be tested individually, but only as a whole. In the case of electrostatics we have electrons with constant mass and charge determining the density of the electric field. The field exerts a force calculated by Coulomb's Law; in the general form, $\mathbf{F}=\rho \cdot \mathbf{E}$. Given the value of this force, we calculate the acceleration of the matter by Newton's Second Law. Weyl concludes:

The laws thus constitute a cycle. ... We require this whole network of theoretical considerations to arrive at an experimental means of verification,- if we assume that what we directly observe is the motion of matter. (Even this can be admitted only conditionally.) We cannot merely test a single law detached from this theoretical fabric! The connection between direct experience and the objective element behind it, which reason seeks to grasp conceptually in a theory, is not so simple that every single statements of the theory has a meaning which may be verified by direct intuition. (Weyl 1922, p. 67)

Now, the argument does not explain why experience, i.e. an 'individual' experience, cannot intervene in the middle and break up the cycle. One may wonder, in other words, what that glue is which holds the cycle together and which prevents observations from falsifying laws in a discrete manner. In the last sentence of the quoted passage Weyl resorts to a version of meaning holism. On that view, abandoning one law impacts on the status of another law just by virtue of changing the meaning of the terms of that latter. But we have not yet seen any justification of it.

\section{Symbolic representation}

Weyl's brief discussion leaves us with an uncertain link between the non-intuitive status of physical laws — or 'laws of nature' — and holism. The version of holism is a Duhemian one. Theories cannot be tested individually, and mathematics remains firmly outside the scope of the empirical. Weyl returns to the same issues nearly forty years later, shortly before his death in Weyl (1954). ${ }^{13}$ Let me summarise its major

13 Other congenial remarks are scattered in Weyl (1949) the bulk of which was published in 1926. 
claims. Physical science progresses towards the ever more increasing symbolisation. And this is to be welcomed. The goal of science is to remove the subjective element of experience. Symbolic constructions allow us doing exactly that. They do not appeal to any unique subjective element in cognition, and they are in principle accessible to every agent to the same extent. Phenomenal qualities are inadequate for understanding the world, since they are unreliable in generating predictions. They contain too many accidental features unique to the given experience. By stripping experience of those phenomenal qualities we arrive at symbolic constructions. They alone provide the required generality, strong enough to give us the predicting tools.

Mathematical physics, according to Weyl, owes its success to just such a transition to symbolic representation. What of mathematical symbolism itself? Its key notion seems to be the notion of a variable. A typical mathematical theorem will have to be general. For example, given any three numbers $a, b$, and $c$, we say that if $a<b$ and $b<c$, then $a<c$. Generality associated with the introduction of variables is interpreted as a possibility of continuing the procedure indefinitely. It is a hypothetical generality: if we can provide an actual construction of indefinitely reproducing the indicated operation, then we are engaging in a typical mathematical activity. And the same generality is present in other purely theoretical activities. Thus, for instance, we construct space as a continuum of possible locations.

The claim has the following form. In science we have to account for phenomena which are not accessible to our immediate experience. Science is occupied with predictions. As such, it must possess generality. And if it has generality, it necessarily requires symbolic constructions involving variables. But there is a second, apparently independent argument to the same effect. If phenomenal qualities are subjective, we must strive to make them objective and subject-invariant. As we push the boundaries of the intuitive and visualisable further, we should ultimately remain with nothing but symbolic constructions. Thus:

Whereas for Huygens colors were 'in reality' oscillations of the ether, they now appear merely as mathematical functions of periodic character depending on four variables that as coordinates represent the medium of space-time. What remains is ultimately a symbolic construction of exactly the same kind as that which Hilbert carries through in mathematics. (Weyl 1949, p. 113)

And there should be no need for tracing the intuitive content of scientific explanations back to our ordinary perceptions. Such a procedure would require a purely qualitative explanation and would not even be intelligible.

Weyl's account contains, then, the following key elements. There is a hierarchy of disciplines. There is a level of 'epistemology', the philosophical enquiry. It supplies us with general principles, such as the principle of no action at a distance. There is a level of mechanics providing us with symbolic, that is, mathematical, constructions devoid of physical content. There is a level of physics that assigns physical meaning to the formulae of mechanics. But there is a hurdle to clear. The field equations themselves are mathematical formulae. Literally put, they cannot tell us what force or any other physical notion is. The empirical content is assigned to them by epistemological principles. Therefore, the latter are called to mediate between pure mathematics and mathematical physics. The idea here, one notices, is remarkably close to Friedman's 
ideas: the choice of the relative a priori for a particular theory is similarly dictated by philosophical considerations. ${ }^{14}$

Given the interrelations between physics, mechanics, and philosophy, the scientific edifice becomes an organic body and holism is a natural outcome. On one hand, physical theories rely on the symbolism of mechanics, on the other-they ultimately follow epistemological assumptions. What of mathematical applicability?

Since science progresses towards objectivity and, that is, towards increased symbolism and formalism, one suspects that the qualitative principles are whittling away in the process. What remains, or in any case should remain, is a symbolic construction of reality. Mathematics will be invading the domain of physics, replacing physical theories with its 'blank forms'. Why would such an intrusion be legitimate in principle? Although never spelled out with clarity, Weyl's ultimate answer is that reality is 'endowed with a structure'. ${ }^{15}$ Structures, described axiomatically, would eventually provide us with a priori knowledge of the world. One feels that this cannot be the end of the story: there may be mutually inconsistent systems describing incompatible structures, and the choice will have to be made by means other than mathematical. Nevertheless the main programmatic claim is clear. Mathematics is applicable to the study of nature, since nature itself has mathematical properties.

\section{Conclusion}

Terminological worries aside, Reichenbach's 1920 theory should, I think, be regarded as a form of conventionalism. So far as they involve an element of arbitrariness and decision, relative a priori statements bear less resemblance to Kant's a priori than Poincaré's conventions. Compared with Poincaré's or even Carnap's original views, considerable modifications are involved. Friedman's interpretation is at its best when it supplies the philosophical rationale for the Folk View and for the continuity in scientific paradigms. But since in attributing synthetic apriority to arithmetic and geometry Kant intends to dismiss the challenge of skepticism, there is a good systematic reason to contrast Kant's a priori with relative a priori. Kant is able to solve the problem of mathematical applicability at the cost of making mathematics obey the nature of human cognition. The theory of relative a priori opens up a gap between the a priori nature of mathematics and the empirical nature of (mathematical) physics. It is, therefore, inept in answering the skeptic.

Weyl's theory, sketchy as it may be, is able to solve the applicability problem, but at no discount price. Its conclusion is a Pythagorean one: mathematics is useful in our theories of nature, since mathematical formalism reflects the deep structure of the world.

Acknowledgements I am grateful to the audiences at LSE and Boğaziçi, and especially to Philip Kitcher, Stathis Psillos, Alan Richardson, and Jon Williamson, for helpful comments on the earlier version of this paper.

\footnotetext{
14 See e.g. (Friedman, 2001, pp. 105-115).

15 See Weyl (1952, p. 144).
} 


\section{References}

Berkovski, S. (2002). Surprising user-friendliness. Logique Et Analyse, 45, 283-297.

Coffa, J. A. (1991). The semantic tradition from Kant to Carnap. Cambridge, MA: Cambridge University Press.

Friedman, M. L. (1983). Foundations of space-time theories. Princeton, NJ: Princeton University Press.

Friedman, M. L. (1999). Reconsidering logical positivism. Cambridge, MA: Cambridge University Press.

Friedman, M. L. (2001). Dynamics of reason. Stanford, CA: CSLI Publications.

Hanna, R. (2001). Kant and the foundations of analytic philosophy. Oxford: Clarendon Press.

Kant, I. (1953). Prolegomena to any future metaphysics. Manchester: Manchester University Press.

Kant, I. (1996). Metaphysical foundations of natural science. In Theoretical philosophy after 1781. Cambridge, MA: Cambridge University Press.

Kripke, S. A. (1971). Identity and necessity. In S. P. Schwartz (Ed.), Naming, necessity, and natural kinds. Ithaca, NY: Cornell University Press.

Kripke, S. A. (1980). Naming and necessity. Oxford: Basil Blackwell.

Poincaré, J. H. (1952). Science and hypothesis. New York, NY: Dover (French edition published in 1905).

Reichenbach, H. (1958). The philosophy of space and time. New York, NY: Dover (German edition published in 1928).

Reichenbach, H. (1965). The theory of relativity and a priori knowledge. Berkeley, CA: University of California Press (German edition published in 1920).

Sivoukhin, D. V. (1979). General physics (Vol. 1). Moscow: Nauka (in Russian).

Steiner, M. (1998). The applicability of mathematics as a philosophical problem. Cambridge, MA: Harvard University Press.

van Fraassen, B. C. (2006). Structure: Its shadow and substance. British Journal for the Philosophy of Science, 57, 275-307.

Weyl, H. (1922). Space-time-matter (4th ed.). New York, NY: Dover (German first edition published in 1918).

Weyl, H. (1949). Philosophy of mathematics and natural science. Princeton, NJ: Princeton University Press.

Weyl, H. (1952). Symmetry. Princeton, NJ: Princeton University Press.

Weyl, H. (1954). Über den Symbolismus der Mathematik und mathematischen Physik. Studium Generale, 6, 219-228.

Weyl, H. (1955). Erkenntnis und Besinnung. Studia Philosophica, 15, 153-171. 